\title{
Real Time Multigraphs for Communication Networks: An Intuitionistic Fuzzy Mathematical Model
}

\author{
Siddhartha Sankar Biswas, Bashir Alam and Mohammad Najmud Doja \\ Department of Computer Engineering, Faculty of Engineering and Technology, \\ Jamia Millia Islamia University, New Delhi-110025, India
}

Received 2013-04-23, Revised 2013-05-23; Accepted 2013-07-17

\begin{abstract}
Many problems of computer science, communication network, transportation systems, can be modeled into multigraphs (or graphs) and then can be solved. Nowadays, the networks are expanding very fast in huge volumes in terms of their nodes and the connecting links. For a given alive network, in many situations, its complete topology may not be always available to the communication systems at a given point of time because of the reason that few or many of its links (edges/arcs) may be temporarily disable owing to damage or attack or blockage upon them and of course they are under repair at that point of time. Such cases are now so frequent that it calls for rigorous attention of the researchers, in particular to those who are concerned with Quality of Service (QoS) while in a network. Even in most of the cases the cost parameters corresponding to its links are not crisp numbers, rather intuitionistic fuzzy numbers (or fuzzy numbers). Thus at any real time instant, the complete multigraph is not available but a submutigraph of it is available to the system for executing its communication or transportation activities. Under such circumstances, none of the existing algorithms on Shortest Path Problems (SPP) can work. In this study the authors propose a mathematical model for such types of multigraphs to be called by 'Real Time Multigraphs' (RTmultigraphs) in which all real time information (being updated every q quantum of time) are incorporated so that the communication/transportation system can be made very efficiently with optimal results. It is a kind of intuitionistic fuzzy mathematical model being the most generalized form of the crisp multigraphs. As a special case, RT-multigraphs reduce to the case of 'RT-graphs'. Finally an intuitionistic fuzzy method is developed to solve the shortest path problem in a RT-Multigraph. As a special case the problem reduces to fuzzy shortest path problem in a RT-Multigraph.
\end{abstract}

Keywords: IFS, IFN, Multigraphs, RT-Multigraphs, RT-Graphs, Neighbor Node, TBL, Link Status, LSV, LSC, TBN, RN, Communicable Node, RT-IF-Min-Weight Arc-Set, IF Shortest Path Estimate, RT IF Relaxation

\section{INTRODUCTION}

Graph theory has wide applications in several branches of Engineering, Science, Social Science, Medical Science, to list a few only out of many. Graph theory has wide applications in several branches of Engineering, in particular in Computer Science, Communication systems, Civil Engineering and also in, science, social science, optimization, management science, medical science, economics to list a few only out of many. The mathematical model 'multigraph' (Biswas et al., 2012) is a generalized concept of graph as multiple links (edges/arcs) may exist between nodes. For example, in a communication model in an Adhoc Network or MANET, multipath features between two adjacent nodes are very common. Two neighboring

Corresponding Author: Bashir Alam, Department of Computer Engineering, Faculty of Engineering and Technology, Jamia Millia Islamia University, New Delhi-110025, India 
routers in a network topology might share more than one multiple direct connections between them (instead of just one), so as to reduce the bandwidth as compared to the case of single connection. Many real life important problems of communication network, transportation network, cannot be modeled into graphs, but can be well modeled into multigraphs. Besides that, in most of the cases of such directed multigraphs, the real data about the weights of the arcs are not always crisp but intuitionistic fuzzy (or fuzzy) numbers. In this study we propose a very generalized notion of 'multigraphs' which is a highly flexible and appropriate model as it incorporates the real time information of the network problem to facilitate the decision maker to search for an efficient and optimized results/solutions. We call such multigraphs by 'Real Time Multigraphs' or 'RTmultigraphs'. Clearly a RT-multigraph is a variable representation of a network with respect to time. We then develop an algorithm to solve the Shortest Path Problem (SPP) in a RT-multigraphs. Consequently, our algorithm may produce different results at different time of start but each time the results will be most efficient for that real time only. Throughout in this study, we consider those multigraphs which are without loops.

\subsection{Preliminaries}

We present a basic preliminaries on the IFS theory of Atanassov (1999) and also on the existing notion of multigraphs (Alam et al., 2013).

\subsection{Intuitionistic Fuzzy Set (IFS)}

The Intuitionistic Fuzzy Set (IFS) theory of Atanassov is now a well known powerful soft computing tool to the world. If $X$ be a universe of discourse, an intuitionistic fuzzy set $\mathrm{A}$ in $\mathrm{X}$ is a set of ordered triplets $\mathrm{A}=\left\{\left\langle\mathrm{x}, \mu_{\mathrm{A}}(\mathrm{x}), \mathrm{v}_{\mathrm{A}}(\mathrm{x})\right\rangle: \mathrm{x} \in \mathrm{X}\right\}$ where $\mu_{\mathrm{A}}, \mathrm{v}_{\mathrm{A}}: \mathrm{X} \rightarrow[0,1]$ are functions such that $0 \leq \mu_{\mathrm{A}}(\mathrm{x})+v_{\mathrm{A}}(\mathrm{x}) \leq 1 \forall \mathrm{x} \in \mathrm{X}$. For each $\mathrm{x} \in \mathrm{X}$ the values $\mu_{\mathrm{A}}(\mathrm{x})$ and $v_{\mathrm{A}}(\mathrm{x})$ represent the degree of membership and degree of nonmembership of the element $\mathrm{x}$ to $\mathrm{A} \subset \mathrm{X}$, respectively and the amount $\pi_{\mathrm{A}}(\mathrm{x})=1$ $\mu_{\mathrm{A}}(\mathrm{x})-v_{\mathrm{A}}(\mathrm{x})$ is called the hesitation part. Of course, a fuzzy set is a particular case of the intuitionistic fuzzy set if $\pi_{\mathrm{A}}(\mathrm{x})=0, \forall \times \in \mathrm{X}$. For details of the classical notion of Intuitionistic Fuzzy Set (IFS) theory, one could see the book (Atanassov, 1999).

The concept of an Intuitionistic Fuzzy Number (IFN) is of importance for quantifying an ill-known quantity. In our work here throughout, we use the notion of triangular Intuitionistic Fuzzy Numbers (IFN) and the basic operations like IF addition $\oplus$, IF subtraction $\Theta$ and 'ranking' of Intuitionistic Fuzzy Numbers (IFNs). Trivially, any crisp real number can be viewed as a fuzzy number (Biswas et al., 2012) or as an IFN. There is no unique method for ranking $n$ number of IFNs, because all the existing methods are soft computing methods. Each method has got merits and demerits depending upon the properties of the application domains and the problem under consideration. However, if $A_{1}, A_{2}, A_{3}, \ldots, A_{n}$ be $n$ IFNs sorted in IF ascending order (in fact it is a kind of non-ascending order, assuming that the IF equal IFNs takes corresponding positions at random if there is no loss of generality) by any good pre-decided method (Mitchell, 2004) i.e., if $\mathrm{A}_{1} \prec \mathrm{A}_{2} \prec$ $\mathrm{A}_{3} \prec \ldots \ldots \prec \mathrm{A}_{\mathrm{n}}$ then $\mathrm{A}_{1}$ and $\mathrm{A}_{\mathrm{n}}$ are called respectively the IF-min and IF-max of these $n$ IFNs. Almost all the existing methods of ranking IFNs were developed independently i.e., not as extensions of the existing methods of ranking of fuzzy numbers. Although several authors have reported several ranking methods of fuzzy numbers, all are having limitations too i.e., not an absolute method suitable for every application domain. However, if $A_{1}, A_{2}, A_{3}, \ldots$, $A_{n}$ be $n$ fuzzy numbers sorted in fuzzy ascending order by a pre-decided method (Allahviranloo et al., 2011) i.e., $A_{1} \prec A_{2} \prec A_{3} \prec \ldots \ldots \prec A_{n}$, then $A_{1}$ and $A_{n}$ are called respectively the fuzzy-min and fuzzy-max of these $n$ fuzzy numbers.

\subsection{Multigraph}

A multigraph $G$ is an ordered pair $(V, E)$ which consists of two sets $V$ and $E$, where $V$ or $V(G)$ is the set of vertices (or, nodes) and $E$ or $E(G)$ is the set of edges (links or arcs). Here, although multiple edges or arcs might exist between pair of vertices but in our discussion in this study we consider that no loop exists (Alam et al., 2013).

Multigraphs may be of two types: undirected multigraphs and directed multigraphs. In an undirected multigraph the edge $(i, j)$ and the edge $(j, i)$, if exist, are obviously identical unlike in the case of directed multigraph. For a latest algebraic study on the theory of multigraphs, the work (Biswas et al., 2012) may be seen.

Figure 1 shows below a directed multigraph $G=(V$, $\mathrm{E})$, where $\mathrm{V}=\{\mathrm{A}, \mathrm{B}, \mathrm{C}, \mathrm{D}\}$ and $\mathrm{E}=\left\{\mathrm{AB}_{1}, \mathrm{AB}_{2}, \mathrm{BA}\right.$, $\mathrm{AD}, \mathrm{AC}, \mathrm{CB}, \mathrm{BD}, \mathrm{DB}\}$.

A multigraph $\mathrm{H}=(\mathrm{W}, \mathrm{F})$ is called a submultigraph of the multigraph $\mathrm{G}=(\mathrm{V}, \mathrm{E})$ if $\mathrm{W} \subseteq \mathrm{V}$ and $\mathrm{F} \subseteq \mathrm{E}$. The Fig. 2 shows a submultigraph $\mathrm{H}$ of the multigraph $\mathrm{G}$. 


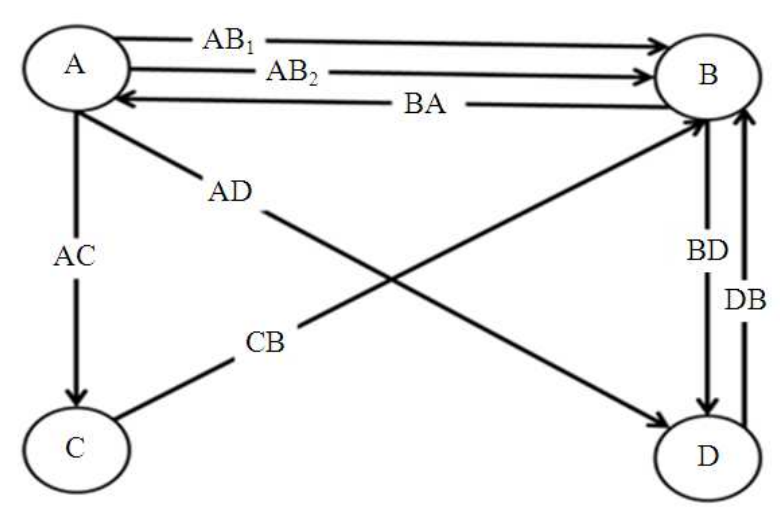

Fig. 1. A multigraph $\mathrm{G}$

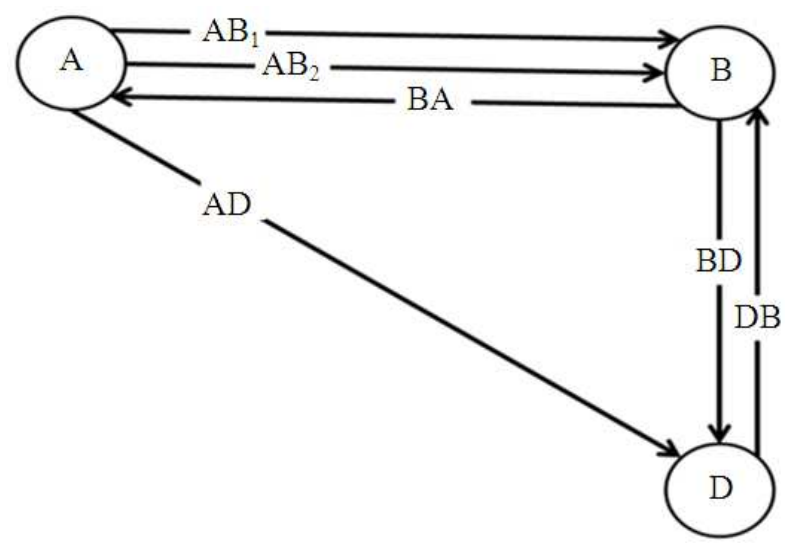

Fig. 2. A submultigraph $\mathrm{H}$ of $\mathrm{G}$

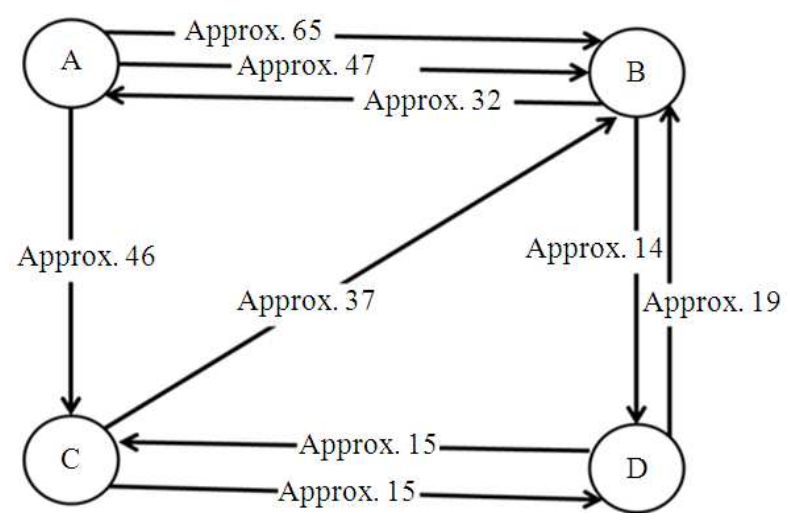

Fig. 3. A multigraph $\mathrm{G}$ with IF weights (IFNs) of arcs

We now consider the modeling of a very real situation of networks and define a generalized notion of multigraphs/graphs.

\subsection{Real Time Multigraph (RT-Multigraph) and Real Time Graph (RT-Graph)}

In most of the real life problems of networks, be it in a communication model or a transportation model, the weights of the arcs are not always crisp but intuitionistic fuzzy numbers (or, at best fuzzy numbers). For example, the Fig. 3 shows a public road transportation model for a traveler where the cost parameters for traveling each arc have been considered as IFN which are the more generalized form of fuzzy numbers involving two independently estimated degrees: degree of acceptance and a degree of rejection.

\subsection{Neighbor' Node}

For a given node $\mathrm{u}$, the node $\mathrm{v}$ will be designated as a 'neighbor' node of $\mathrm{u}$ if $\mathrm{u}$ has at least one link from $\mathrm{u}$ to $\mathrm{v}$.

In our work here we consider more real situations which are actually and frequently faced by the present communication systems. For example, consider an Adhoc Network or a MANET where there may exist multiple paths between two neighbor nodes, but because of some reasons one or more number of paths may be temporarily damaged and hence temporarily unavailable for transmission of packets by a node $u$ to its neighbor node $\mathrm{v}$. This is very useful information to the communication system if available to the sender node in advance.

\section{Example 3.1}

Consider the following directed Multigraph $G$ with IF weights. If the node A knows well in advance that the link AC (weight = 3) and the link AB (weight $=10$ ) are temporarily unavailable, then the node A has no other option but to send the its packets to its neighbor $\mathrm{B}$ via the path $\mathrm{AB}$ (weight $=15$ ) only.

In our proposed mathematical model of multigraphs, we incorporate the real time data from the network to make the multigraphs more dynamic, more useful and hence more efficient to the users. Every node of the multigraph carries an information vector corresponding to each of its neighbor nodes. If the node $\mathrm{u}$ has the node $\mathrm{v}$ as a neighbor node then $\mathrm{u}$ carries the following information handy with it:

- $\quad$ Suppose that there are $n(\geq 1)$ number of links from $u$ to $\mathrm{v}$ outward which are $\mathrm{uv}_{1}, \mathrm{uv}_{2}, \ldots \ldots, \mathrm{uv}_{\mathrm{n}}$. Let $\mathrm{u}$ designate them as $1 \mathrm{st}, 2 \mathrm{nd}, 3 \mathrm{rd}, \ldots . . ., \mathrm{nth}$ 


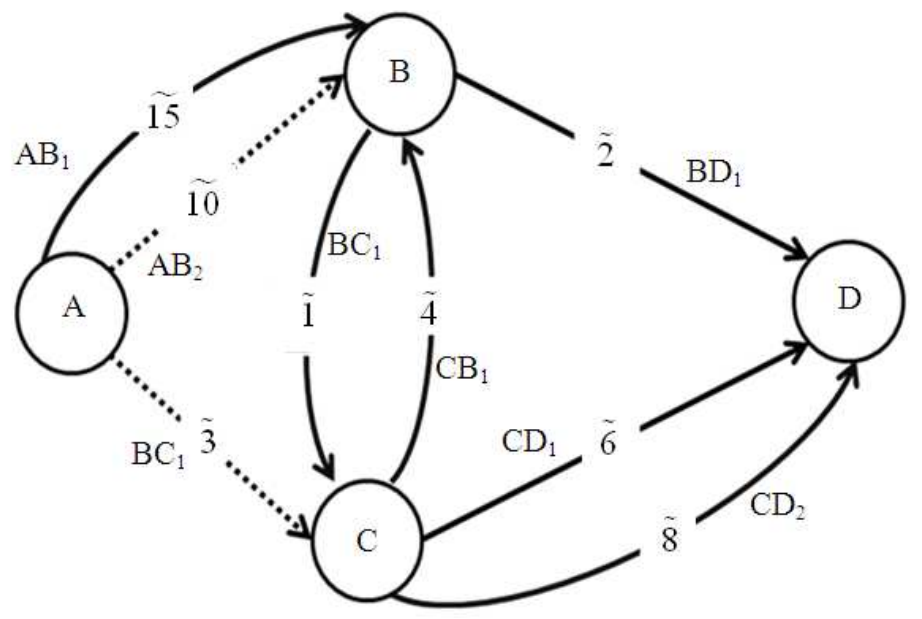

Fig. 4. A Multigraph G having few links damaged temporarily

- In real life situation, because of natural phenomenon (flood, earthquake, thunderstorm, solar storm) or because of some kind of external attack or technical failure or because of an predictable/unpredictable damage of the link, (to list a few only out of many such type of real life problems), it may happen in reality that during a period of time the rth link $\mathrm{uv}_{\mathrm{r}}$ of the node $\mathrm{u}$ to its neighbor $\mathrm{v}$ is non-functional $(\mathrm{r}=1$, $2,3, \ldots, n)$, as shown in Fig. 4. In our proposed model, this is precious information and is available with the node $u$ here in advance

\subsection{Link Status Vector (LSV)}

Corresponding to every neighbor node $\mathrm{v}$, there exist a Link Status Vector (LSV) $I_{u v}=\left(i_{1}, i_{2}, i_{3}, \ldots\right.$. , $i_{n}$ ) of $u$, where at any given point of time $i_{r}$ takes any of the two values from $\{0,1\}$ for $r=1,2,3, \ldots, n$ with the following significance:

$$
\begin{aligned}
\mathrm{i}_{\mathrm{r}} & =0, \text { if the link } \mathrm{uv}_{\mathrm{r}} \text { is non-functional. } \\
& =1 \text {, if the link } \mathrm{uv}_{\mathrm{r}} \text { is functional. }
\end{aligned}
$$

\subsection{Temporarily Blocked Link (TBL) and Link Status}

If at a given time $i_{r}$ happens to be 0 , i.e., if the link $u_{r}$ is non-functional then we say that the link $u v_{r}$ is a temporarily blocked link (tbl) from $u$. The value $i_{r}$ is called the 'link status' of the link $\mathrm{uv}_{\mathrm{r}}$.

In real situation the complete multigraph thus may not be available due to existence of non-functional status for few links, i.e., due to existence of few tbls and consequently a sub-multigraph of it be available for communication (Example: for communication of packets in an Adhoc Network/ MANET, or for a salesman to travel many cities, or for a buss/truck carrying goods/passengers in a transportation network).

If a node $u$ has $k(\geq 0)$ number of neighbor nodes $v 1$, $\mathrm{v} 2, \mathrm{v} 3, \ldots \ldots, \mathrm{vk}$, then $\mathrm{u}$ carries $\mathrm{k}$ number of LSV: $\mathrm{I}_{\mathrm{uv} 1}, \mathrm{I}_{\mathrm{uv}}$, $\mathrm{I}_{\mathrm{uv} 3}, \ldots \ldots, \mathrm{I}_{\mathrm{uvk}}$. In our mathematical model, we propose that there is a system $\mathrm{S}$ for the multigraph which updates all the information vectors of all the nodes after every quantum time $\tau$. This quantum $\tau$ is fixed (can be reset) for the system $\mathrm{S}$ a multigraph but different for different multigraphs, in general depending upon the various properties of the physical problem for which a multigraph is modeled.

\subsection{Link Status Class (LSC)}

For a given node $u$, the collection of all LSV are called 'Link Status Class' (LSC) of u denoted by $\mathrm{I}_{\mathrm{u}}$. If a node $\mathrm{u}$ has $\mathrm{k}(\geq 0)$ number of neighbor nodes $\mathrm{x} 1, \mathrm{x} 2$, $\mathrm{x} 3, \ldots ., \mathrm{xk}$, then:

$$
\mathrm{I}_{\mathrm{u}}=\left\{\mathrm{I}_{\mathrm{ux} 1}, \mathrm{I}_{\mathrm{ux} 2}, \mathrm{I}_{\mathrm{ux} 3}, \ldots, \mathrm{I}_{\mathrm{uxk}}\right\}
$$

\subsection{Temporarily Blocked Neighbor and Reachable Node}

If $v$ is a neighbor node of a given node $u$ and if $I_{u v}$ is a null vector at a given instant of time then $v$ is called a temporarily blocked node (tbn) of $\mathrm{u}$ for that instant.

However, since it is a temporary phenomenon and if any of the links be repaired in due time, then obviously a 'blocked neighbor' may regain its 'neighbor' status at some later stage. 


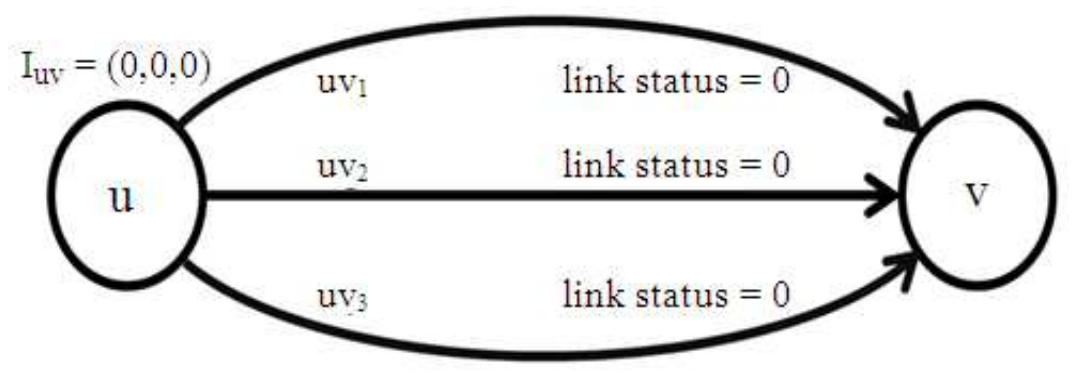

Fig. 5. A TBN $v$ of the node $u$

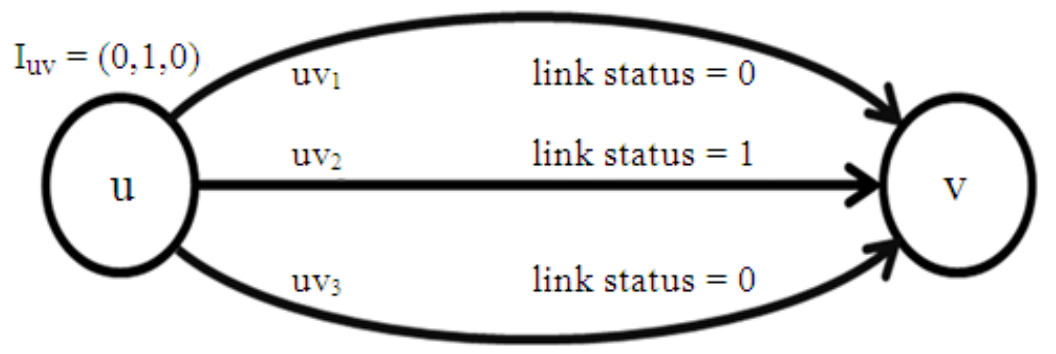

Fig. 6. A rn v of the node $u$

If a neighbor $v$ is not a tbn, then it is called a reachable neighbor (rn) of u. For example, Fig. 5 and 6 show a tbn and a rn respectively.

\subsection{Communicable Node}

For a given node $u$, if $I_{u} \neq \varphi$ and at least one member of $I_{u}$ is non-null at a given point of time, then the node $u$ is called a communicable node for that instant of time.

If $u$ does not have any neighbor node then $I_{u}=\varphi$ and in that case it is trivial that further communication is never possible. However, if $\mathrm{I}_{\mathrm{u}} \neq \varphi$ and all the members of $I_{u}$ are null vectors at a point of time, then it signifies that further communication is not possible temporarily.

All the real time information mentioned/defined above will get automatically updated at every node of the multigraph at every q quantum of time (for a quantum $q$ to be pre-fixed depending upon the properties of the network, on what kind of communication/transportation it is performing).

We call such type of multigraphs by 'Real Time Multigraphs' or 'RT-multigraphs' as they contain real time information of the networks. Consequently, for a given network the RT-multigraph is not a static multigraph but changes with time. As a special case, if a network can be modeled into a graph then we call our proposed model as 'Real Time Graph' or 'RT-graph'.
We now develop a method to find an intuitionistic fuzzy shortest path from a source node to a destination node in a RT-multigraph.

\subsection{If Shortest Path in a RT-Multigraph}

Mukherjee (2012), Sathi used a heuristic methodology for solving the IF shortest path problem using the Intuitionistic Fuzzy Hybrid Geometric (IFHG) operator, with the philosophy of Dijkstra's Algorithm. In Karunambigai et al. (2007) in a team work with Atanassov, developed a method based on dynamic programming to find the shortest paths in intuitionistic fuzzy graphs. Gani (2010) also developed a method on searching intuitionistic fuzzy shortest path in a network. But multigraphs now-a-days a very important model of network because there are many real life problems of network, transportation, communication, circuit systems, which cannot be modeled into graphs but into multigraphs only. There is no attempt made so far in the literature for searching an IF shortest path in a multigraph. The dynamic model "RT-multigraph' is a new concept on the generalization of multigraphs or graphs considering its huge potential for real time applications in communication or transportation systems. 


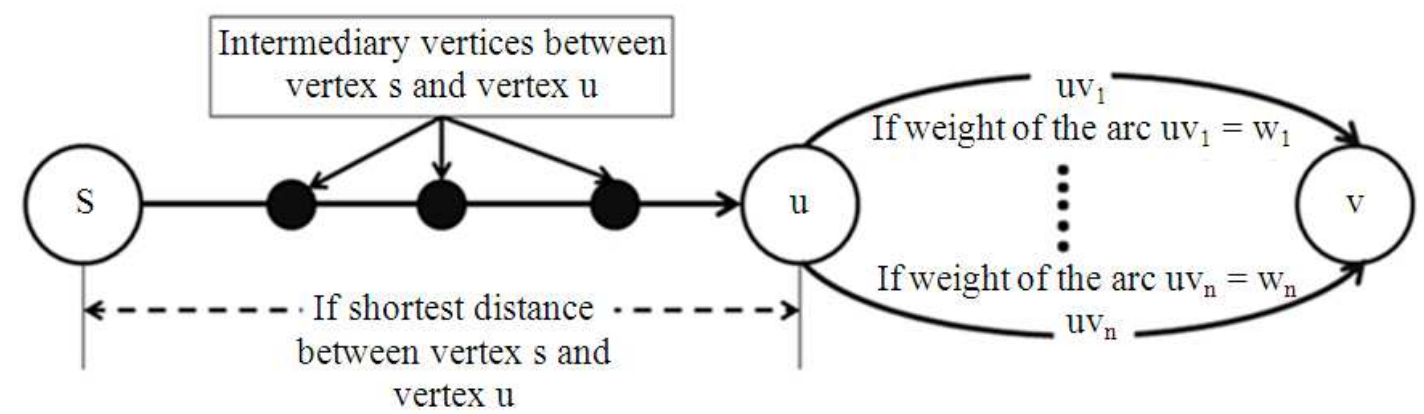

Fig. 7. Estimation procedure for $\mathrm{d}[\mathrm{v}]$

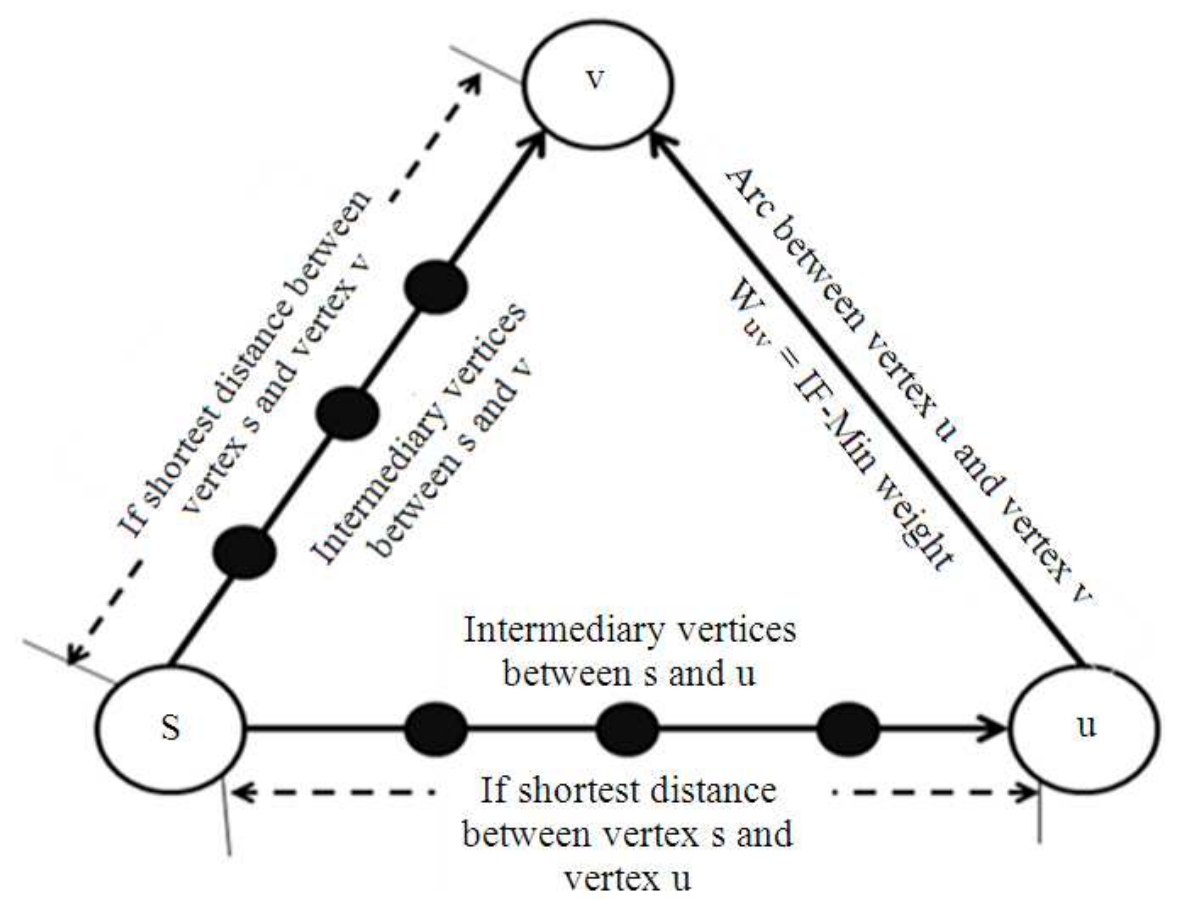

Fig. 8. Diagram showing how the RT-IF-RELAX algorithm works in a RT-multigraph

We solve the SPP for RT-multigraphs where we also use the notion of Dijkstra's Algorithm but with simple softcomputations without using any hybrid geometric operators, using only basics of Atanassov's IFS operators (Atanassov, 1999). For this, first of all we need to define the terms: RTIF-Min-Weight arc-set, IF shortest path estimate $(\mathrm{d}[\mathrm{v}])$ of a vertex, RT-IF relaxation of an arc, in the context of the RTmultigraphs and then develop few subalgorithms.

\subsection{RT IF-Min Weight Arc-Set of a Directed RT-Multigraph}

Consider a directed RT-multigraph $\mathrm{G}$ where the arcs are of IF weights (IFNs). Suppose that $v$ is a rn of $u$ in $G$ with $\mathrm{I}_{\mathrm{uv}}=\left(\mathrm{i}_{1}, \mathrm{i}_{2}, \mathrm{i}_{3}, \ldots . ., \mathrm{i}_{\mathrm{n}}\right)$ corresponding to $\mathrm{n}$ number of $\operatorname{arcs} \mathrm{uv}_{1}, \mathrm{uv}_{2}, \ldots \ldots, \mathrm{uv}_{\mathrm{n}}$ from the vertex $\mathrm{u}$ to the vertex $\mathrm{v}$, where $\mathrm{n}$ is a non-negative integer.

Consider the set $\mathrm{S}_{\mathrm{uv}}$ whose elements are the arcs from the vertex $u$ to the vertex $v$ for each of which the link status is 1 and let $\mathrm{W}_{\mathrm{uv}}$ be the same set $\mathrm{S}_{\mathrm{uv}}$ but keyed and sorted in non-descending order by the value of their respective IF weights, using a suitable predecided ranking method of IFNs.

With no loss of generality, let us suppose that $\mathrm{W}_{\mathrm{uv}}$ $=\left\{\left(u_{1}, w_{1 u v}\right),\left(u_{2}, w_{2 u v}\right),\left(u_{3}, w_{3 u v}\right), \ldots .,\left(u_{v r}\right.\right.$, $\left.\left.\mathrm{w}_{\text {ruv }}\right)\right\}$, where the rest (n-r) number of links have the status 0 for each. 
If two or more number of IF weights are equal then they may appear at random at the corresponding place of non-descending array in our discussion.

If there is no confusion, let us denote the multiset $\left\{\mathrm{w}_{1 \mathrm{uv}}, \mathrm{w}_{2 \mathrm{uv}}, \mathrm{w}_{3 \mathrm{uv}}, \ldots . . ., \mathrm{w}_{\mathrm{ruv}}\right\}$ also by the same name $\mathrm{W}_{\mathrm{uv}}$. Let $\mathrm{w}_{\mathrm{uv}}$ be the IF-min value of the multiset $\mathrm{W}_{\mathrm{uv}}=$ $\left\{\mathrm{w}_{1 \mathrm{uv}}, \mathrm{w}_{2 \mathrm{uv}}, \mathrm{w}_{3 \mathrm{uv}}, \ldots \ldots . ., \mathrm{w}_{\mathrm{ruv}}\right\}$. Clearly, $\mathrm{w}_{\mathrm{uv}}=\mathrm{w}_{1 \mathrm{uv}}$, as the multiset $\mathrm{W}_{\mathrm{uv}}$ is already sorted by a pre-choosen IFN ranking method.

Then the set $\mathrm{W}=\left\{<(\mathrm{u}, \mathrm{v}), \mathrm{w}_{\mathrm{uv}}>:(\mathrm{u}, \mathrm{v}) \in \mathrm{E}\right.$ and $\mathrm{v}$ is a rn of $\mathrm{u}\}$ is called the RT IF-Min-weight arc-set of the RT-multigraph G. Suppose that the subalgorithm IFMWA (G) returns the RT IF-Min-weight arc-set W.

\subsection{IF Shortest Path Estimate $d[v]$ of a rn Vertex $v$ in a Directed RT-Multigraph}

Suppose that $\mathrm{s}$ is the source vertex and the currently traversed vertex is $u$. At this real time we check whether $\mathrm{u}$ is a communicable node or not. If yes, then we proceed further. There may exist multiple arcs between from the vertex $u$ to the rn vertex $v$. If $v$ is not a rn, then $d[v]$ need not be estimated. Otherwise, using the value of $\mathrm{w}_{\mathrm{uv}}$ from the RT-IF-min weight multiset $\mathrm{W}$ of a directed RTmultigraph, we can now find the IF shortest path estimate i.e., $\mathrm{d}[\mathrm{v}]$ of any $\mathrm{rn}$ vertex $\mathrm{v}$, in a directed RTmultigraph as below:

(IF shortest path estimate of vertex $v)=($ IF shortest path estimate of vertex $u$ ) $\oplus$ (IF-Min of all the IF weights corresponding to the arcs from the vertex $u$ to the vertex $v$ )

$$
\text { Or, } \mathrm{d}[\mathrm{v}]=\mathrm{d}[\mathrm{u}] \oplus \mathrm{w}_{\mathrm{uv}} \text { (as shown in Fig. 7) }
$$

\subsection{IF Relaxation of an Arc}

We extend the classical notion of relaxation to the case of IF weighted arcs. At this real time the important deviation from the classical method is that we do not attempt to do relaxation of an arc/link if it is a tbl. By IF relaxation we shall mean here the relaxation process of an arc whose weight is an IFN. For this, first of all we initialize the multigraph along with its starting vertex and IF shortest path estimate for each vertices of the multigraph G. The following 'IF-INITIALIZATIONSINGLE-SOURCE' algorithm will do:

\section{IF-Initialization-Single-Source (G, s):}

1. For each vertex $v \in \mathrm{V}[\mathrm{G}]$

$$
\begin{array}{lr}
\text { 2. } & \mathrm{d}[\mathrm{v}]=\infty \\
\text { 3. } & \mathrm{v} \cdot \boldsymbol{\pi}=\mathrm{NIL} \\
\text { 4. } & \mathrm{d}[\mathrm{s}]=0
\end{array}
$$

After the IF initialization, the process of IF relaxation of each arc begins, but to be applicable at real time only and hence tbl cases are excluded from the execution of relaxation jobs. The sub-algorithm RT-IFRELAX, as shown in Fig. 8, plays the vital role to update $d[v]$ i.e., the IF shortest distance value between the starting vertex $\mathrm{s}$ and the $\mathrm{rn}$ vertex $\mathrm{v}$ (which is neighbor of the current traversed vertex $\mathrm{u}$ ).

\section{RT-IF-RELAX (u, v, W):}

1. IF $\mathrm{u}$ is a communicable node, THEN

2. IF $\mathrm{v}$ is a rn, THEN

3. $\quad$ IF d $[\mathrm{v}] \prec \mathrm{d}[\mathrm{u}] \oplus \mathrm{w}_{\mathrm{uv}}$

4. THEN d $[\mathrm{v}] \leftarrow \mathrm{d}[\mathrm{u}] \oplus \mathrm{w}_{\mathrm{uv}}$

5. $\quad$ v. $\pi \leftarrow u$

where, $\mathrm{w}_{\mathrm{uv}} \in \mathrm{W}$ is the IF-Min weight of the arcs from vertex $u$ to vertex $v$ and $v$. $\pi$ denotes the parent node of vertex $\mathrm{v}$.

\subsection{Real Time IF Shortest Path Algorithm (RT-IFSP Algo) in a RT-Multigraph}

We now present our main algorithm to find single source IF shortest path in a RT-multigraph (Fig. 9).

We name this by 'Real Time Intuitionistic Fuzzy Shortest Path Algorithm' i.e., in short by the title RTIFSPA. In this algorithm we use the above subalgorithms and also the subalgoritm EXTRACT-IFMIN (Q) which extracts the node $\mathrm{u}$ with minimum key using IF ranking method and updates $\mathrm{Q}$ at real time.

\section{RT-Ifspa (G, s):}

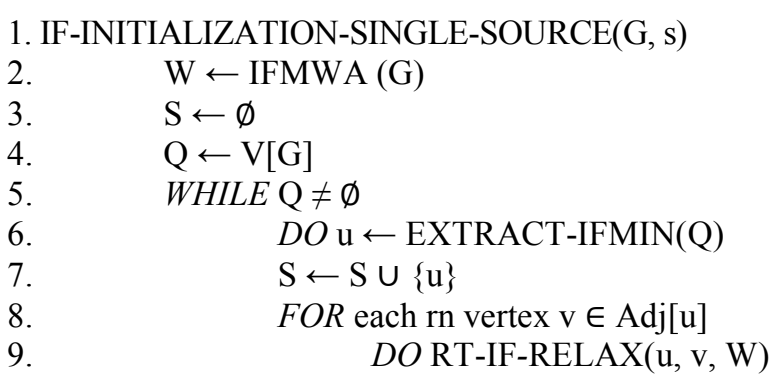

\section{Example 4.1:}

Consider the following directed RT-Multigraph G where the IF weights are shown against each link. We want to solve the single-source IF shortest paths problem taking the vertex $A$ as the source vertex and the vertex $\mathrm{D}$ as the destination vertex, where and the LSCs of all the nodes of the RT-multigraph $G$ are $I_{A}$, $\mathrm{I}_{\mathrm{B}}, \mathrm{I}_{\mathrm{C}}$ and $\mathrm{I}_{\mathrm{D}}$ given by: 


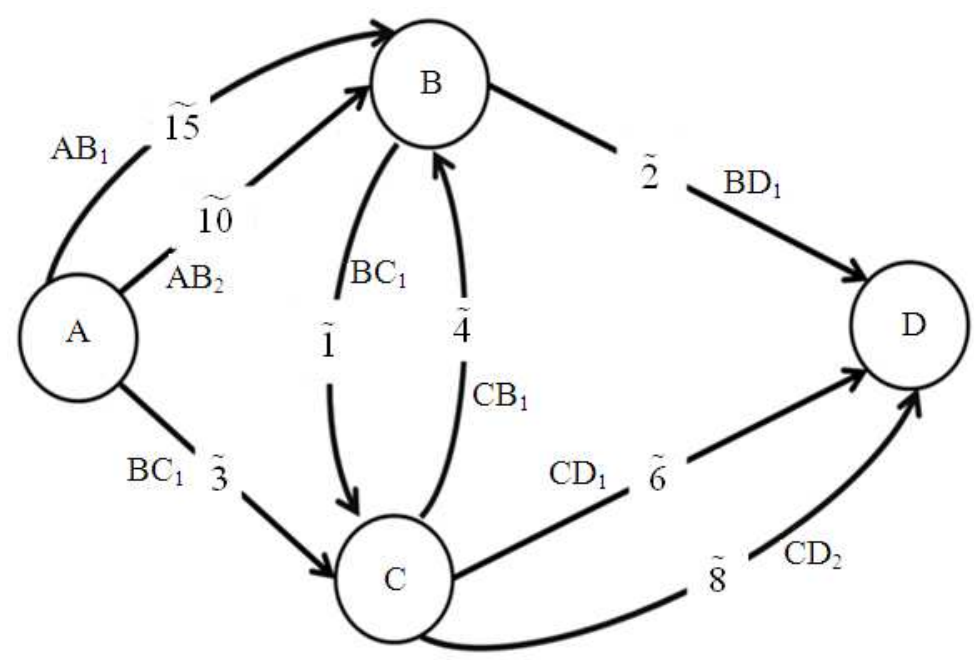

Fig. 9. A RT-multigraph G

$\mathrm{I}_{\mathrm{A}}=\left\{\mathrm{I}_{\mathrm{AB}}, \mathrm{I}_{\mathrm{AC}}\right\}$ where $\mathrm{I}_{\mathrm{AB}}=(0,1), \mathrm{I}_{\mathrm{AC}}=(1)$;

$\mathrm{I}_{\mathrm{B}}=\left\{\mathrm{I}_{\mathrm{BC}}, \mathrm{I}_{\mathrm{BD}}\right\}$ where $\mathrm{I}_{\mathrm{BC}}=(1), \mathrm{I}_{\mathrm{BD}}=(1)$ and

$\mathrm{I}_{\mathrm{C}}=\left\{\mathrm{I}_{\mathrm{CB}}, \mathrm{I}_{\mathrm{CD}}\right\}$ where $\mathrm{I}_{\mathrm{CB}}=(1), \mathrm{I}_{\mathrm{CD}}=(1,0)$

Clearly the RT-IFSPA algorithm computes to yield the following result:

- $\mathrm{w}_{\mathrm{AB}}=10, \mathrm{w}_{\mathrm{AC}}=\tilde{3}, \mathrm{w}_{\mathrm{CB}}=\tilde{4}, \mathrm{w}_{\mathrm{CD}}=\tilde{6}$ and $\mathrm{w}_{\mathrm{BD}}=\tilde{2}$; and then

- $\mathrm{S}=\{\mathrm{A}, \mathrm{C}, \mathrm{B}, \mathrm{D}\}$, i.e., the IF shortest path from the source vertex $A$ is:

$$
\mathrm{A} \rightarrow \mathrm{C} \rightarrow \mathrm{B} \rightarrow \mathrm{D}
$$

- d-values i.e., IF shortest distance estimate-values of each vertex from the starting vertex $\mathrm{A}$ is:

$$
\begin{gathered}
\mathrm{d}[\mathrm{A}]=0, \mathrm{~d}[\mathrm{C}]=\operatorname{IFN} \tilde{3}, \mathrm{~d}[\mathrm{~B}]=\operatorname{IFN} \tilde{7} \\
\mathrm{~d}[\mathrm{D}]=\operatorname{IFN} \tilde{9}
\end{gathered}
$$

\subsection{Fuzzy Shortest Path in a RT-Multigraph}

If the hesitation part is zero for each member in IFS, it automatically reduces to a fuzzy set. A fuzzy set is a trivial case of IFS. Consequently, the IF methods and algorithms reduce to the fuzzy methods as special cases which are presented below.

Consider a directed RT-multigraph $\mathrm{G}=(\mathrm{V}, \mathrm{E})$ with fuzzy weights (fuzzy numbers). Suppose that $\mathrm{s}$ is the source vertex and the currently traversed vertex is $u$. At this real time we check whether $u$ is a communicable node or not. If yes, then we proceed further. There may exist multiple arcs between from the vertex $u$ to the $r n$ vertex $v$. If $v$ is not a rn, then $d[v]$ need not be estimated. Otherwise, using the value of $\mathrm{w}_{\mathrm{uv}}$ from the RT-Fuzzymin weight multiset $\mathrm{W}$ of $\mathrm{G}$, we can now find the fuzzy shortest path estimate i.e., $d[v]$ of any rn vertex $v$, in a directed RT-multigraph as below.

$\therefore \mathrm{d}[\mathrm{v}]=($ Fuzzy shortest distance between $\mathrm{s}$ and $\mathrm{u})$ $\oplus$ (Fuzzy-Min of all the fuzzy weights corresponding to the arcs from the vertex $u$ to the vertex $v$ ).

By RT-fuzzy relaxation, we shall mean here the relaxation process, to be executed at real time only, of an arc whose weight is a fuzzy weight. As in case of intuitionistic fuzzy cases, we first initialize the RTmultigraph along with its starting vertex and fuzzy shortest path estimate for each vertices of the RTmultigraph G.

\section{Fuzzy-Initialization-Single-Source (G, s):}

1. FOR each vertex $v \in V[G]$

2. $\mathrm{d}[\mathrm{v}]=\infty$

3. $\quad$ v. $\pi=\mathrm{NIL}$

4. $\mathrm{d}[\mathrm{s}]=0$

The sub-algorithm RT-F-RELAX, plays the vital role to update $\mathrm{d}[\mathrm{v}]$.

\section{RT-F-Relax (u, v, W):}

1. IF $\mathrm{u}$ is a communicable node, THEN

2. IF $\mathrm{v}$ is a rn, THEN 
5.

$\mathrm{V} . \pi \leftarrow \mathrm{u}$

Finally, we present the algorithm for 'Real Time Fuzzy Shortest Path' which we call by RT-FSPA.

\section{RT-FSPA (G, s):}

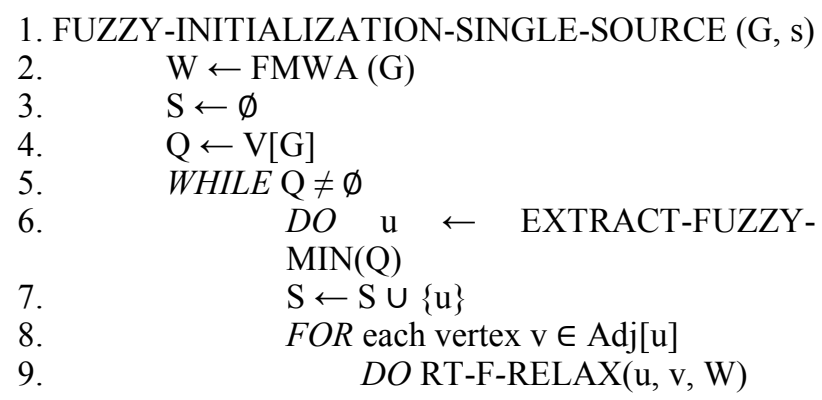

\section{CONCLUSION}

Multigraph is a mathematical model which is a generalization of graph. There are many real life problems of network, transportation, communication, circuit systems. Which cannot be modeled into graphs but into multigraphs only. In real life situations, in many of these directed multigraphs, the weights of the arcs are not always crisp but intuitionistic fuzzy (or fuzzy). Besides that, due to some reason few links may be temporarily unavailable to the communication system. Consequently, the complete topology of the multigraph of a network may not be available to the system but a submultigraph of it. In this study we make a mathematical modeling of such real time status of a network by introducing a generalized structure called by 'RT-multigraph' or 'RT-graph'. The real time data/information are updated at each node of a the RTmultigraph at every quantum time q. We also develop a method to find IF shortest path from a source vertex to a destination vertex of a directed RT-multigraph. As a special case, the method reduces to the method of finding fuzzy shortest path (Klein, 1991) in a directed RT-multigraph. However, the notion of RT-graphs is a trivial case of RT-multigraphs and hence all the algorithms of RT-multigraphs can well be applicable to the case of RT-graphs too.

\section{REFERENCES}

Alam, B., S.S. Biswas and M.N. Doja, 2013. Fuzzy shortest path in a directed multigraph. Eur. J. Sci. Res., 101: 333-339.

Allahviranloo, T., S. Abbasbandy and R. Saneifard, 2011. A method for ranking of fuzzy numbers using new weighted distance. Math. Comput. Applic., 16: 359-369.

Atanassov, K., 1999. Intuitionistic Fuzzy Sets: Theory and Applications. 1st Edn., Physica-Verlag, Heidelberg, New York, ISBN-10: 3790812285, pp: 323 .

Biswas, S.S., B. Alam and M.N. Doja, 2012. A theoretical characterization of the data structure 'multigraph'. J. Contemporary Applied Math., 2: 88-106.

Gani, A.N., 2010. On searching intuitionistic fuzzy shortest path in a network. Applied Math. Sci., 4: 3447-3454.

Karunambigai, M.G., P. Rangasamy, K. Atanassov and N. Palaniappan, 2007. An intuitionistic fuzzy graph Fmethod for finding the shortest paths in networks. Adv. Soft Comput., 42: 3-10. DOI: 10.1007/978-3-540-72434-6 1

Klein, C.M., 1991. Fuzzy shortest paths. Fuzzy Sets Syst., 39: 27-41. DOI: 10.1016/01650114(91)90063-V

Mitchell, H.B., 2004. Ranking-intuitionistic fuzzy numbers. Int. J. Unc. Fuzz. Knowl. Based Syst., 12: 377-386. DOI: 10.1142/S0218488504002886

Mukherjee, S., 2012. Dijkstra's algorithm for solving the shortest path problem on networks under intuitionistic fuzzy environment. J. Math. Model. Algorithms, 11: 345-359. DOI: 10.1007/s10852012-9191-7 